\title{
Foreword
}

\section{Orthopedic Anesthesia}

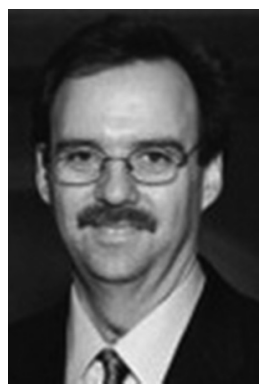

Lee A. Fleisher, MD

Consulting Editor

In the context of health care reform, it is increasingly important for anesthesiologists to demonstrate their value in patient care. One of the areas that has received a great deal of attention in that anesthesiologists impact the quality and costs of care is in orthopedic surgery. With the development of regional anesthesia and increasing interest in assessing outcomes after orthopedic surgery, anesthesiologists have taken a leadership role in delivering value. Much of the success of the Perioperative Surgical Home is in the area of orthopedic anesthesia. In this issue of Anesthesiology Clinics, a selection of excellent articles highlights the care paradigms and outcomes in this population.

In choosing guest editors for this issue, I solicited Nabil M. Elkassabany, MD, MSCE, Assistant Professor of Anesthesiology and Critical Care at the University of Pennsylvania Perelman School of Medicine, and Edward R. Mariano, MD, MAS, Associate Professor of Anesthesiology at Stanford University School of Medicine. Dr Mariano is also Chief of Anesthesiology and Perioperative Care Service and Associate Chief of Staff for the Inpatient Surgical Services. Both of these individuals have led Divisions of Regional Anesthesia and have formal training in clinical research and therefore have the expertise to ensure that the care paradigms are both evidence-based and lead to better outcomes. Both of them have also published original articles and reviews in the area of regional anesthesia and orthopedic surgery. Together they have solicited an outstanding group of contributors, including themselves, to provide us with the most up-to-date information.

Lee A. Fleisher, MD Perelman School of Medicine University of Pennsylvania Philadelphia, PA 19104, USA

E-mail address:

Lee.fleisher@uphs.upenn.edu 